\title{
La conversión social de Santa Mónica en una ciudad sustentable
}

Guillermo Ibarra. Universidad Autónoma de Sinaloa, Culiacán, México. Adriana Moreno. Universidad Autónoma de Sinaloa, Culiacán, México.

Resumen | Santa Mónica, California, inició su transición hacia un sistema de planeación urbana sustentable a finales de la década de 1970, continuándolo hasta la actualidad. El punto de partida del fenómeno fue un movimiento ciudadano surgido en 1978, que se consolidó en 1981 como la principal fuerza política local, al obtener por primera vez la mayoría de los asientos en el Consejo de la Ciudad. Este trabajo analiza los factores propulsores del plan local de desarrollo sustentable en Santa Mónica. Asimismo, explica cómo se estructuró el andamiaje político que fue eficaz para traducir la negociación de los diferentes actores sociales entre los paradigmas de la competitividad urbana y el de desarrollo sustentable con justicia social. Se concluye que una ciudad sustentable es un ente social en medio de permanente conflicto y negociación, y que su éxito depende del compromiso y habilidad de los ciudadanos y las instituciones de gobierno para darles curso.

PALABras CLAVE | desarrollo sustentable, desarrollo urbano, marketing urbano, planificación urbana.

ABSTRaCT | Santa Monica, California, began its transition towards a sustainable urban planning in late 1970s, continuing to the present. The starting point was a citizens' movement emerged in 1978, which was consolidated in 1981 as the major political force when they obtained, for the first time, most of the seats on the City Council. This paper examines the driving forces behind the local project of sustainable development in Santa Monica. Also, it explains how the political platform that was effective to interpret the negotiation of social actors between the paradigms of urban competitiveness and sustainable development with social justice was structured. The general conclusion is that a sustainable city is a social construction in the midst of constant conflicts and negotiations, and that its success depends on the commitment of citizens and government institutions to make it possible.

KEY WORDs | sustainable development, urban development, urban marketing, urban planning.

Recibido el 3 de agosto de 2011, aprobado el 7 de diciembre de 2012

E-mail: Guillermo Ibarra, gibarra@uas.edu.mx | Adriana Moreno, adriana.moreno@uas.edu.mx 


\section{Introducción}

Este ensayo explica cómo se instituyó en Santa Mónica una estrategia de desarrollo local sustentable desde 1981, cuando una coalición progresista asumió el poder municipal, hasta 2010, con la reinauguración del Centro Comercial "Santa Monica Place” y la entrada en vigor del plan Land Use and Circulation Element (LUCE). Responderemos las siguientes preguntas: ¿Qué factores políticos e institucionales posibilitaron el inicio en Santa Mónica de una estrategia de desarrollo sustentable? ¿Cuáles son las características socioculturales que dieron soporte a la formalización de ese proyecto urbano? ¿Qué relación guardan sus políticas de sustentabilidad urbana y las de competitividad económica territorial? ¿Cuáles son las contradicciones que existen entre la planeación urbana sustentable y los compromisos de justicia social y espacial?

Al analizar la experiencia de Santa Mónica, cuestionamos que sea la implementación de principios de planeación, diseño urbano y tecnologías "verdes" lo que forja una ciudad sustentable, y, por el contrario, sostenemos que se afinca en factores socioculturales y políticos. Coincidimos con Swyngedouw (1997) en visualizar la sustentabilidad como un sistema "híbrido de formas sociales, económicas, políticas y ecológicas que continuamente se articulan y se re-articulan, según sus especificidades espaciales e históricas" (p. 142). Así, la configuración de ciudades sustentables va más allá de una planeación instrumental, pues constituye tanto una forma urbana como una forma social, incluyendo una visión política y un ideal social. Ligada a los aspectos técnicos, la proyección de ciudades sustentables implica gestionar una "colección compleja de ideas, discursos, prácticas, materiales y conflictos políticos a través de los cuales son producidas" (Whitehead, 2003, p. 1186), pues son una realidad geohistórica estructurada entre conflictos y negociaciones, protagonizados por actores sociales en un marco institucional definido.

Por efecto de la reciente globalización capitalista, los cambios en la cultura política y las nuevas ideas sobre gobernanza y administración pública han provocado, a nivel mundial, que los Estados nación compartan su poder con gobiernos de espacios territoriales más pequeños y con estructuras más amplias (Pal, 2006), lo que influye en la planeación urbana. Sin demeritar el valor heurístico que tiene la categoría de gobernanza para analizar la interacción y resolución de conflictos en las sociedades urbanas, optamos en este ensayo por ubicarnos en otra perspectiva cercana, enfocándonos en las características socioculturales del lugar, desde la geografía comprehensiva, pues el discurso de la sustentabilidad es parte de un imaginario urbano en donde el individuo o los grupos construyen espacialidades al vivir su sociabilidad.

La gobernanza refiere estilos de gobierno donde se confunden las fronteras entre los sectores público y privado. Para Benz (2007), la gobernanza "está mayormente asociada con el diseño de las políticas más allá del Estado, con cooperación entre los actores públicos y privados, o la propia regulación cooperativa privada” (p. 5). Esa cooperación es proteica, fluye a través de diferentes estructuras, redes, jerarquías, competencias o espacios de negociación, que conducen a la coordinación e interacción de actores, guiada por reglas explícitas o implícitas. El poder gubernamental aparece 
como el "tejedor" de la red a través de la cual se interactúa y configura la agenda local (Schuppert, 2007, p. 39). Esta gobernanza concierne a la regulación en un contexto de pluralidad de ideas y confluencia de intereses, lo que Arthur Benz (2007) llama "arenas conectadas". Al transitarse de formas de gobierno a gobernanza en múltiples escalas territoriales y a través de dominios funcionales diversos, el papel del aparato estatal se orienta a asegurar la factibilidad de proyectos económicos y sociales patrocinados cada vez más multilateralmente (Pike, Rodríguez \& Tomaney, 2011).

El Programa de Naciones Unidas para los Asentamientos Humanos (United Nations Human Settlements Programme [un-Habitat]] (2009) caracteriza los retos de la planeación urbana sustentable partiendo de las preguntas: ¿cómo deben distribuirse los beneficios del desarrollo urbano? y ¿cómo se logra el equilibrio entre derechos individuales y preocupaciones colectivas conforme avanza este desarrollo? Responde que existen diferentes trayectorias para procurarlo, por lo conflictivo de integrar un sistema de acción colectiva en escenarios sociales cambiantes. De esta manera, la gobernanza refleja las formas en que los territorios urbanos se organizan para lograrlo, definiéndose como:

La suma de las formas en que las personas e instituciones, públicas y privadas, planifican y gestionan los asuntos comunes de la ciudad. Es un proceso continuo a través del cual los conflictos o diversos intereses se ajustan y dan lugar a la cooperación. Incluye instituciones formales, así como acuerdos informales y capital social de los ciudadanos (un-Habitat, 2007, p. 73) ${ }^{1}$.

La búsqueda de formas de gobernanza para la toma de decisiones en el marco de la administración del territorio es una reacción pos-crisis, un paradigma emergente de estrategias para orientar el desarrollo urbano hacia formas sustentables e incluyentes, con gobierno de redes (gobierno relacional, multinivel o facilitador), mediante la cooperación de actores (Aguilar, 2006). Frente al empresarialismo neoliberal impuesto en los espacios urbanos por el capitalismo global (Harvey, 1989), surge un discurso alternativo, "neoestructural" o "neomoderno" (Barton, 2006), apoyado en esquemas profundos de gobernanza.

El concepto es atractivo y sugerente, aunque puede ser problemático, pues presupone una especie de ciudadanía con niveles de identidad social que difícilmente existen. Barton (2006) sostiene que "para explicar por qué algunas ciudades han sido vanguardistas y otras más reticentes en la incorporación de los criterios de sustentabilidad en su planificación, debemos remitirnos a aspectos de conciencia de gobernanza, de pensamiento visionario de líderes claves (principalmente en la administración pública), y también aludir a la sensibilidad frente a la presión de la sociedad civil y la capacidad de respuesta frente a los compromisos multilaterales" (p. 34). Pero, ¿a qué "sensibilidad" es posible aludir, o a qué criterio de "visionario" se puede apelar en una sociedad urbana multiclasista, desigual, con ciudadanías excluyentes? (Isin, 2002). No hay solución técnica; ignorarlo implica desconocer que los individuos en la ciudad ostentan diferentes identidades cartográficas por

1 Las citas que no cuentan con una versión en español han sido traducidas directamente del inglés por los autores. 
su peculiar inserción en la división social del trabajo en sentido amplio (Harvey, 2007), y configuran conocimientos geográficos específicos de referencia para actuar socialmente, mapas mentales que organizan sus vidas en el espacio. En una ciudad se gestan múltiples identidades cartográficas; por ejemplo, los empresarios inmobiliarios tienen un conocimiento territorial de la ciudad que les permite competir en el mercado del suelo, la vivienda y la construcción en general. Existe una identidad cartográfica dominante o de un grupo hegemónico que impone en el territorio sus valores y conocimiento sobre la ciudad; que configura su propia personalidad mediante una diferenciación social frente al resto y determina la forma de actuar y conocer la economía y urbanización como moralmente deseables. Si soslayamos esa multiplicidad de identidades ciudadanas, entonces podríamos incurrir en un voluntarismo romántico acerca de la planeación urbana sustentable, como si fuese una praxeología donde una mecánica de negociación tiene como base una "visión común" de la ciudad (lo cual no impide que compartamos un sentido común como seres sociales, pero es insuficiente). Por estos argumentos, en este artículo intentamos otra explicación (no necesariamente excluyente) sobre la búsqueda de sustentabilidad en una ciudad, reconociendo que una aproximación con base en el concepto de gobernanza podría ser también apropiada si se asumiese, como lo considera Barton (2006), un "proceso permanente" hacia la construcción de una ciudad deseable en términos sociales y ambientales. Sin embargo, hay una doble deformación en esto. La primera, por considerar la posibilidad de predecir el arribo de un tipo de sociedad gracias a un "proceso" voluntario, lo que relega el significado particular de los hechos concretos y se convierte en fin en sí mismo, redundante. Segundo, por asumir que la historia humana se puede "construir" como si se manufacturase un objeto. Arendt (1995) critica que la inversión que realizó Marx de la teoría de Hegel sobre la historia llevó a una corriente teórica (y política) a considerarla como una totalidad coherente, regida por leyes, con una existencia material, independiente de la conciencia que los seres sociales tuvieran de ella. De ello presumen, según Arendt, que si se conociesen esos mecanismos del funcionamiento social, se podría actuar, para acelerar, retardar o desviar su curso. De esta forma terminó considerándose a la sociedad como un objeto de fabricación, una realidad moldeable a los deseos del hombre, a condición de actuar conforme a supuestas leyes inmanentes englobadas en procesos. Los defectos de este historicismo y voluntarismo otorgan artificialmente a la historia una previsibilidad de la cual carece. Arendt agrega que la historia tiene muchos principios, pero ningún fin, en el sentido de que la acción humana, al ser libre, tiene el imperativo de la aleatoriedad. La misma autora señala que el actor social no sabe realmente lo que está ocurriendo cuando actúa; solo una vez que están concluidos los hechos, o cuando un historiador los estudia, surge una "historia", una narrativa de esos acontecimientos acabados. Asimismo, el principio de una "historia" se puede entender en circunstancias en que ocurren episodios reveladores, que conectan el presente con ciertos fenómenos previos aprehendidos en ese momento revelador mediante una retrospectiva, pero esto no significa que ese presente puede replicarse a través de un principio identificado como causa del análisis. En ciencias sociales y en la política es algo polémico considerar la posibilidad de "construir" una ciudad sustentable (a la manera en que quiso construir 
el "socialismo", supuestamente con base científica, sin éxito), como si fuera la fabricación de un artefacto, y menos verosímil es que tengamos, como en el caso de la manufactura, instrumentos, mecanismos y reglas para darle existencia. El instrumentalismo que se les otorga a los mecanismos de planeación sustentable por medio de la gobernanza proviene, en nuestra opinión, de ese historicismo y de esa distorsión ideológica. El concepto se convierte en una nueva panacea para lograr cierta concertación de voluntades que lleven a construir una realidad social deseada; en este caso, una ciudad sustentable.

Nuestro acercamiento al problema parte de la formulación de la cuestión urbana por Lefebvre (1972), Harvey (2007) y Castells (2004), pioneros de un enfoque que llevó a la reaserción de la geografía en el entendimiento de la vida social, antes hegemonizada por visiones temporalistas de la historia. En esa corriente de pensamiento, Soja (2000) propone recuperar el poder explicativo de lo espacial por medio de lo que denominó "consequential geographies". Percibe como "una dialéctica socioespacial" la influencia mutua y relación formativa entre la dimensión social y espacial de la vida humana, cada una dando forma a la otra por vías similares. Por consecuencia, cualquier tema de análisis puede abordarse como relaciones y desarrollo social, tanto procesos sociales que configuran y dan significado a las geografías humanas como espacialidades en las cuales vivimos. "Estas dos ideas juntas -sostiene- ayudan a comprender el significado de las consequential geographies, una asertiva perspectiva espacial y del poder explicativo del pensamiento espacial" (p. 4). En esta noción, las personas somos tanto seres temporales como espaciales; ambas experiencias son mutuamente correspondientes y formativas, en términos explicativos y conductuales, por lo que no tiene sentido otorgar a priori mayor importancia a un aspecto que a otro:

De esa manera, desde que construimos nuestras geografías multiescalares, de manera más poderosa que otros, podemos actuar para cambiarlas o reconfigurarlas, y así incrementar o disminuir sus efectos positivos o negativos. Esos esfuerzos para modificar las configuraciones espaciales de nuestra existencia -ya sea que ello implique redecorar nuestras casas, luchar en contra de la segregación racial en nuestras ciudades, crear políticas para reducir la disparidad del ingreso entre países desarrollados y subdesarrollados o combatir el calentamiento global- no implican asumir inocentemente objetivos universales. Estos esfuerzos son objetivo y fuente de propósitos conflictivos, fuerzas en competencia y acciones políticas polémicas a favor o en contra del statu quo. El espacio no es un ente vacío. Está siempre lleno de política, ideología y otras fuerzas que dan forma a nuestras vidas y nos desafían para involucrarnos en conflictos sobre la geografía (Soja, 2000, p. 19).

Para comprender por qué Santa Mónica fue un lugar propicio para la adopción de un proyecto de desarrollo urbano sustentable, analizaremos el carácter del lugar, el armazón sociocultural de las ciudades, su capital político, fortaleza institucional e interacciones entre los principales grupos de interés. A la par, revisaremos el despliegue de tecnologías sustentables y prácticas de planeación y diseño de la ciudad. Nuestro estudio se apoyó en documentos oficiales y fuentes secundarias. Es también resultado de un trabajo de campo realizado en el verano de 2010, con información 
recabada de entrevistas a actores clave de la comunidad, como Shannon Parry, coordinadora del Plan de Sustentabilidad; Terry O’ Day, fundador de la organización no gubernamental Environment Now (Medio ambiente ahora) y miembro del Consejo de la Ciudad; y Ana Jara, integrante del consejo directivo de los Santa Monicans for Renters' Rights (smrr, Residentes de Santa Mónica en Defensa de los Derechos de Inquilinos) y activista de la asociación de residentes del vecindario Pico.

\section{Antecedentes}

Santa Mónica, una ciudad de 90.750 habitantes, forma parte del Gran Los Ángeles, la segunda conurbación más grande de Estados Unidos, que incluye los condados de Orange, Los Ángeles, San Bernardino, Ventura y Riverside, tiene una población de casi 18 millones y abarca más de 340 ciudades (California Department of Finance, 2009) (véase Figura 1).

\section{FIgura 1 | Localización de Santa Mónica en California y Los Ángeles}

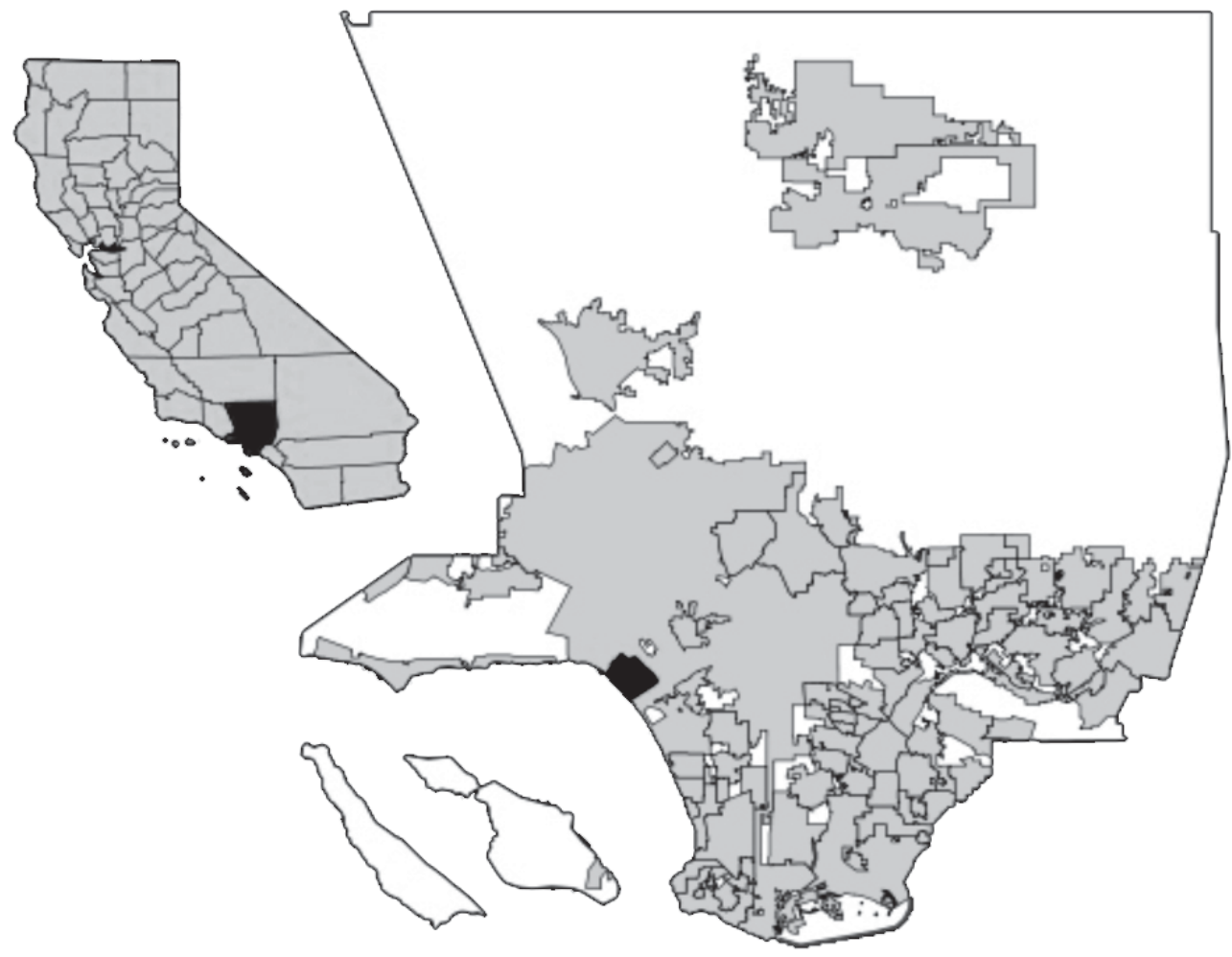

FUENTE HTTPS://EN.WIKIPEDIA.ORG/WIKI/FILE:LA_COUNTY_INCORPORATED_AREAS_SANTA_ Monica_HIGHLIGHTED.SVG

A pesar de ser un fragmento de ese tejido megalopolitano, Santa Mónica posee una historia distintiva, con tradiciones, carácter cultural e instituciones particulares que posibilitan estudiarla como configuración socioespacial específica. Su territorio ha pertenecido a tres naciones: en 1542, a Espańa; a México, a partir de 1822, cuando 
logró independizarse de Nueva España; y a Estados Unidos desde 1848, al finalizar la guerra de invasión contra México (1845-1848). Santa Mónica era una ranchería costera sin cambios notables hasta la "fiebre del oro" (1848-1855), cuando arribaron masivamente a California aventureros de otros lugares y países. En poco tiempo, Los Ángeles se convirtió en un centro de comercio, juego, crimen y violencia. Sus familias residentes, enlazadas de diversas maneras a Los Ángeles, fueron afectadas por los recién llegados (Keil, 1998).

Con una población de apenas 417 habitantes en 1880, el turismo ya era importante para la economía de Santa Mónica. Esta tendencia se reforzó con la llegada del ferrocarril Southern Pacific en 1885, su incorporación como municipalidad del Condado de Los Ángeles en 1886, y la apertura del monumental Arcadia Hotel en 1887. En 1899 recibió cerca de 200.000 visitantes y al pasar el siglo tenía 3.057 habitantes y 15.252 en 1920 (Scott, 2004). De 1921 a 1975, con la apertura de la Douglas Air Craft Company, la industria aeronáutica fue otro factor impulsor del desarrollo (Pitt, 1997). En la década de 1970, el Consejo de la Ciudad favoreció el crecimiento inmobiliario. El auge del negocio de bienes raíces fue notorio; viviendas y edificios eran demolidos, relocalizados o sustituidos por departamentos de lujo, para acelerar la expansión económica (Scott, 2004). Paradójicamente, ese desarrollismo pergeñó un fuerte activismo social progresista. Con el nacimiento del movimiento SMRR en 1978 y su triunfo en las elecciones locales de 1979 y 1981, se iniciaron profundas transformaciones políticas. En los comicios de 1979, la asociación logró la aprobación de una Ley de Control de Arrendamiento y la elección de dos de sus miembros, William H. Jennings y Ruth Yannatta Goldway, como integrantes del Consejo de la Ciudad. Desde el poder municipal, el movimiento forjó un régimen progresista en asuntos tanto sociales como medioambientales, desplegando un radicalismo de clase media (Kann, 1986). A mediados de la década de 1990, este activismo urbano y la solidez fiscal del ayuntamiento favorecieron una política sistemática de protección medioambiental, en un contexto internacional propicio.

Con la Cumbre de Río en 1992, el desarrollo sustentable ganó consenso mundial. Fue entonces cuando el ayuntamiento de Santa Mónica conformó la Comisión de Medio Ambiente, que integró el concepto al Programa de la Ciudad Sustentable (Santa Monica Sustainable City Program), aprobado por el Consejo de Gobierno en septiembre de 1994. Con ello se formalizó una nueva agenda de desarrollo urbano, como sucedía en San José, California; Jacksonville, Florida; Portland, Oregon; Seattle, Washington; y Cambridge, Massachusetts (Portney, 2003). Aunque la mayoría de los residentes, políticos y funcionarios de Santa Mónica profesan la protección medioambiental, sus políticas avanzan a través de complejas negociaciones, pues los objetivos "duros" de protección de los derechos ciudadanos, vivienda asequible y una mejor calidad de vida, se contraponen con un empresarialismo urbano que usa la sustentabilidad como plataforma política para ganar competitividad interurbana. El gobierno local, en colaboración con la Cámara de Comercio y el Consejo de Turismo de la Ciudad, convirtieron el programa de sustentabilidad en sostén de la mercadotecnia del lugar y la ejecución de proyectos de revitalización urbana orientados a la atracción de visitantes, inversionistas y nuevos residentes de altos ingresos. 
Los principios para el diseño de la ciudad sustentable se fundamentan en el nuevo urbanismo, el crecimiento inteligente y la certificación de edificios verdes -Leadership in Energy and Environmental Design (Certificación LEED: Liderazgo en Diseño Ambiental y Energético) - conformando lo que Farr (2008) denomina "urbanismo sustentable", que implica reducción del uso del automóvil, desarrollo compacto, aumento de áreas verdes, aplicación de tecnología amigable con el medio ambiente, entre otras. Al implementarlas, Santa Mónica obtuvo mejoras en sus indicadores de manejo de los recursos naturales. En 2009, el uso del agua se redujo de 12,6 a 11,9 millones de galones diarios, un descenso del 5 por ciento. En 1999-2000, disminuyó 5,7 por ciento su huella ecológica, que calcula el área de terreno necesario para sostener los patrones de consumo y desechos generados por una comunidad.

Smith (2002), Ellis (2002) y Larsen (2005) coinciden en que este urbanismo sustentable justifica la revitalización neoliberal y la restitución del poder de la clase más poderosa sobre la ciudad. Tiene como base social "alianzas público-privadas" para promover el empresarialismo, es decir, proyectos de innovación cultural, mejora física, infraestructura (parques recreativos, centros comerciales) y la organización de eventos culturales y de negocios (conciertos, exposiciones y festivales locales) (Harvey, 2007). Con tal estrategia y promocionando su imagen de ciudad sustentable, Santa Mónica adquirió una reputación de "lugar innovador, interesante, creativo y seguro para visitar, vivir y consumir” (Harvey, 1989, p. 377), logrando atractividad para la inversión y el turismo. Muestra de ello es la inversión de más de 2,5 millones de dólares que The Marcerich Company realizó en la remodelación del Santa Monica Place reinaugurado en 2010.

Lo anterior tiene como contraparte movimientos contra la injusticia espacial que afecta a barrios vulnerables como Pico y otros de clase media, capaces de organizarse para darles seguimiento a aquellos ideales de la ciudad sustentable que se cimentaron con el movimiento progresista de los ańos setenta.

\section{Desarrollo urbano sustentable}

El desarrollo sustentable busca compatibilizar el crecimiento económico con las aspiraciones de bienestar de la población, y contrarrestar el deterioro social y medioambiental inherente al crecimiento urbano (Moffatt, 1999). En un principio, la visión ambientalista fue promovida por el Banco Mundial, enfocándose hacia los problemas globales de la degradación del medio ambiente, sin referencia a una escala geográfica específica (Aguado, Barrutia \& Etxebarria, 2006). Con la incorporación del espacio, su caracterización descendió al ámbito local (Bithas \& Christofakis, 2006) y se reformuló desde diferentes ideologías e intereses, usándose "para significar casi cualquier cosa que uno desee, lo que constituye parte de su atractivo" (O’Connor, 2002, p. 27).

En el contexto urbano, el desarrollo sustentable asumió un carácter fecundo por los rasgos socioeconómicos y culturales que caracterizan la ciudad (Bithas \& Christofakis, 2006), convirtiéndose en el principal elemento para vivir de "manera sustentable en el planeta" (Nelson, 2001, p. 742). De este modo, en cada urbe, el 
desarrollo sustentable recibió un significado distinto según los aspectos de mayor relevancia para su caso particular. En Estados Unidos, más de veinte ciudades fueron pioneras en sustentabilidad urbana: Santa Mónica, Portland, Seattle, San Francisco, Chattanooga, Jacksonville, Orlando, Tampa, Olympia, Milwaukee, San José, Santa Bárbara, Austin, Indianápolis, Boulder, Cambridge, Boston, Brookline, Scottsdale, Phoenix, Tucson, Brownsville, Cleveland y New Haven. Asimismo, el desarrollo sustentable influye en el diseño de las ciudades en otras regiones del mundo, como en el Reino Unido, Suecia, Holanda, China, Turquía y México.

Para denominar a esta agenda, se han utilizado numerosos conceptos: planeación para un desarrollo urbano sustentable (Naess, 2001); planeación sustentable (Naess, 2001; Wheeler, 2003); sustentabilidad urbana (Lieberherr-Gardiol, 2009); ciudad sustentable (Portney, 2003; Whitehead, 2003); forma urbana sustentable (Bentivegna et al., 2002; Wheeler, 2003); proyecto urbano sustentable (Lieberherr-Gardiol, 2009); desarrollo sustentable de los asentamientos humanos (Deb, 1998); desarrollo urbano sustentable (Bentivegna et al., 2002) y urbanismo sustentable (Farr, 2008).

Kriken (2010), Beatley (2001) y Farr (2008) proponen que la sustentabilidad urbana depende del ensayo de técnicas, tecnologías y pautas de planeación amigables con el medio ambiente. Para Bentivegna et al. (2002), el desarrollo urbano sustentable es "el proceso de adaptar el medio construido a través del tiempo de modo que sostenga patrones de vida, trabajo y producción más prudentes con la naturaleza” (p. 90). Según Naess (2001), la planeación para un desarrollo urbano sustentable se orienta a disminuir el uso de energía y de emisiones de bióxido de carbono per cápita; minimizar el socavamiento de áreas naturales, ecosistemas y terrenos para la producción de alimentos; disminuir el ruido, la contaminación y aumentar las áreas verdes para mantener el lazo emocional de la población con la naturaleza. Según Douglas Farr (2008), el urbanismo sustentable comprende el uso de nuevas tecnologías, estrategias y principios de planeación para rediseñar el medio ambiente construido y modificar los efectos negativos del crecimiento tradicional; para ello sugiere construir calles amigables con el peatón y los ciclistas, manejo eficiente de aguas pluviales, promocionar formas alternativas de transporte, uso de biocombustible, producción local de alimentos, centros multimodales de transportación, techos verdes, jardines comunitarios, ventilación natural, vivienda asequible, desarrollo integral de vecindarios y de terceros lugares como espacios informales de reunión en donde la gente se conoce, interactúa y forma asociaciones: café, biblioteca, lavandería, parque, iglesia. Estas acciones difícilmente pueden concretarse sin otros procesos sociales, correspondientes a los patrones de comportamiento sociocultural, fortaleza de su andamiaje institucional, el papel de los actores sociales, sus interrelaciones y la calidad de los procesos de negociación entre los grupos de interés.

La experiencia de Santa Mónica muestra tradiciones locales de protección medioambiental que pautaron su devenir. Esto puede explicarse con los conceptos de Molotch, Freudenburg y Paulsen (1997), aquellos relativos a carácter y tradición del lugar. En los lugares se generan ciertas tradiciones que persisten a lo largo del tiempo, a pesar de los cambios en su estructura socioespacial y cultural. Esta repetición ocurre, según los autores, a través de un proceso en que nuevos rasgos en la estructura social de un lugar logran transmitirse (lash up) de un contexto de 
organización social a otro, denotando una forma peculiar de interacción entre sus actores e instituciones con el ambiente y el espacio construido, que lo distinguen de otros territorios y forjan un nuevo carácter del lugar. No es este un proceso lineal, sino que ocurre en medio de ajustes y conflictos sociales de diferente índole.

En Santa Mónica, las valoraciones de los diferentes actores locales respecto a la planeación tienen gran peso. Con su proceder moldean el proyecto de la ciudad sustentable, apareciendo desacuerdos sobre qué tipo de sustentabilidad esperan, quién lo decide y a quién beneficia. Cada grupo tiene intereses y percepciones al respecto, a veces divergentes. El gobierno, los empresarios, las organizaciones no gubernamentales y los movimientos de base poseen capacidades y medios para influir en su diseño, y aunque la administración local tiene facultades legales para impulsar políticas, los otros actores moldean el proceder gubernamental suscitando acciones que podrían favorecer más a una parte que a otra, o traducir con mayor eficacia los intereses de un grupo, dependiendo del contexto.

La conjunción de estos procesos de planeación con el activismo social y cultural despliega en Santa Mónica una cierta conflictividad entre actores urbanos, la cual muestra que una ciudad sustentable es un espacio social complejo que cobra forma y función en una condición urbana peculiar, a partir de las capacidades locales de colaboración y solución de conflictos en marcos institucionales.

La condición urbana es una compleja interacción entre sociedad y espacio donde el individuo y la colectividad confluyen, creando una forma física peculiar de la ciudad. Teóricamente, la ciudad es concebida de diversas formas: como un laboratorio social donde el hombre se construye a sí mismo como ser civilizado y racional, construcción que está condenado a transformar permanentemente (Park, 1999); como un complejo social estructurado y organizado (Mumford, 2002); una nueva forma de organización humana que revoluciona la psicología colectiva e individual; un caldero de creatividad (Jacobs, 1971; Rykwert 2002); la proyección total de la sociedad en el espacio, portadora de una capacidad revolucionaria (Castells, 2004; Harvey, 1989; Lefebvre, 1972); la forma suprema de la espacialidad humana (Soja, 2000). En las ciudades surgen imaginarios, identidades colectivas e individuales y diferentes formas de vivir y sentir. Por su praxis cotidiana diferenciada y los distintos contextos institucionales en que actúan, los múltiples grupos sociales construyen identidades cartográficas que difieren entre sí respecto al conocimiento geográfico que adquieren de la ciudad (Harvey, 2007; Isin, 2002). Lo anterior distingue múltiples acciones colectivas con variable grado de coherencia, y las más intensas pueden configurar movimientos sociales o "particularismos militantes" que denotan que todas las políticas tienen su origen en el desarrollo colectivo de "una visión política determinada, por parte de personas determinadas, en tiempos y lugares determinados" (Harvey, 2007, p. 206). Las instituciones y el Estado operan, con mayor o menor eficacia, como mecanismo traductor de las diferentes aspiraciones y demandas de estos particularismos, resultando hegemónicos algunos de ellos.

Estos movimientos son determinantes en la gestión urbana; actúan mediante protestas directas, litigios y actividades concretas de protección al medio ambiente (Souza, 2006). En Santa Mónica se formó un particularismo militante que pudo hegemonizar la planeación urbana con esta orientación. 
Los habitantes de Santa Mónica habían participado esporádicamente en los asuntos locales; dos ejemplos de ello son la asociación Save Our Pier Forever (Salvemos nuestro muelle para siempre), que en 1972 se constituyó para luchar en contra de la demolición del muelle de la ciudad; y la organización, en 1974, de The Airport Neighbors Forum (El foro de los vecinos del aeropuerto), para regular los vuelos del aeropuerto local. En las últimas tres décadas, las organizaciones ciudadanas se han fortalecido frente a los decretos del gobierno local que contradicen sus aspiraciones. Su intervención desempeña una función transformadora en materia social y medioambiental. Podemos fijar el inicio de este fenómeno en 1978, cuando se formó la asociación SMRR, en respuesta a los efectos nocivos del auge inmobiliario. En Santa Mónica se derribaron residencias para construir condominios y departamentos, cuyos precios ya no fueron asequibles para la mayoría de los residentes (Fulton, 1997). Ante esta situación, un grupo de adultos mayores inició un movimiento de inquilinos con el apoyo de activistas del barrio de Ocean Park y de un conjunto de arrendatarios que no podían comprar casa o costear altos alquileres. El despliegue de fuerzas en apoyo a la SMRR y a favor de la Ley de Control de Arrendamiento creó una conciencia de participación ciudadana cuando "muchas personas en Santa Mónica no sabían ni siquiera en qué ciudad vivían" (Fulton, 1997, p. 32). Con apoyo de la ciudadanía, en abril de 1979 la Asociación logró su primer triunfo en los comicios locales. Dos de sus miembros fueron elegidos como integrantes del Consejo de la Ciudad y se aprobó una Ley de Control de Arrendamiento (la más estricta del país), que instituyó un Consejo de Control de Alquileres elegido popularmente (Thorton, 2011; Santa Monica Rent Control Charter Amendment, 2012). La Ley consta de 20 capítulos, en los cuales resaltan los siguientes ítems: ajustes anuales al valor de los alquileres (cap. 3); regulación del monto total que se cobra por unidad de alquiler (cap. 4); designación de los servicios incluidos como parte del alquiler e indemnización si dichos servicios se eliminan o reducen (cap. 8); desalojos permitidos solo por "causas justas" (cap. 9); y normalización del retiro de unidades de vivienda controladas del mercado de alquiler (cap. 5). La aprobación de esta ley y la llegada de la coalición progresista al gobierno local fueron determinantes para estructurar en Santa Mónica un nuevo carácter local e iniciar una tradición de activismo y participación ciudadana en pro de la sustentabilidad.

El éxito del grupo progresista se reafirmó en las elecciones de 1981 cuando las fuerzas de izquierda política y los Renters' Rights (organización en pro de los derechos de los inquilinos) obtuvieron cuatro asientos más en el Consejo de la Ciudad, anteriormente ocupados por banqueros, abogados, propietarios y representantes de los arrendadores e inversionistas, quienes hacen de la ciudad una "maquinaria urbana del crecimiento" (Molotch, 1976, p. 309). En esta ocasión, Jim Conn, Ken Edwards, Delores Press y Dennis Zane se unieron a Ruth Yanatta Goldway en el Consejo, siendo esta última elegida alcaldesa. Con ello, el movimiento de base democrática se convirtió en la principal fuerza política local y, desde entonces, Santa Mónica se diferenció del resto de las ciudades del suroeste de California, exceptuando West Hollywood, por la promoción de justicia social. 
Aunque el nacimiento de la coalición respondió al control del arrendamiento, su agenda se diversificó incluyendo asistencia a los sin casa, discapacitados y adultos mayores; construcción de vivienda asequible y, en general, control del crecimiento (SMRR, 2008). Sin embargo, con el transcurso de los ańos, el régimen progresista se inclinó hacia un proyecto menos radical de competitividad urbana y marketing de la clase empresarial, particularmente del sector turístico (Riposa, 2004).

Visto en perspectiva, en Santa Mónica se conjugaron diversos elementos que generaron una reconfiguración socioespacial (un lash up, Molotch, 1997) de naturaleza distinta a los que le habían precedido, dando lugar a una nueva tradición de corte progresista y responsabilidad medioambiental; entre ellos, el arribo de matrimonios jóvenes integrados por activistas en los diversos movimientos sociales y contracultura de los sesenta; la consolidación del movimiento progresista en 1979, con su triunfo en las elecciones locales; y la formulación y ejecución del Santa Monica Sustainable City Plan (Plan urbano sustentable de Santa Mónica) en 1994, creado para "mejorar el manejo local de los recursos naturales, prevenir el daño al medioambiente y procurar el bienestar social y económico de la comunidad" (Santa Monica Sustainable City Plan, 2003). El carácter, la tradición del lugar y el armazón sociocultural que empezaron a conformarse desde 1970, abrieron paso a una agenda de sustentabilidad que orientó sus patrones de comportamiento político, actitudes críticas de los ciudadanos, estilos de vida alternativos y conciencia ambiental, configurando un capital político que transformó la organización y administración del sistema urbano.

\section{Activismo ciudadano}

En este contexto, irrumpió un protagonismo de diversos actores urbanos para gestionar abiertamente sus intereses. Esta cultura política de cooperación y negociación para la sustentabilidad se correlaciona con el alto perfil económico y educativo de la población de Santa Mónica. En 2010, de los habitantes mayores de 25 años, 95,1 por ciento tenía bachillerato terminado y 64 por ciento, licenciatura o posgrado. La mayoría de raza blanca no hispana, jóvenes adultos profesionales de clase media/media alta, y con el doble del ingreso promedio del resto del condado, 58.933 dólares per cápita, mientras que en el condado de Los Ángeles era de apenas 28.222 dólares. No obstante su alto perfil socioeconómico, más de 70 por ciento de los habitantes de Santa Mónica no cuenta con casa propia.

Según el censo de 2011, el valor promedio de una vivienda en Santa Mónica es de 987.300 dólares, mientras que en el estado de California es de 421.600 dólares; en la ciudad de Los Ángeles, de 421.600 dólares; y en Culver City, de 623.100 dólares (United States Census Bureau, 2011). Esto explica la formación de movimientos ciudadanos de reforma social, como el que impulsó la Ley de Control de Arrendamiento y el proyecto de la ciudad sustentable, con fuertes demandas desde abajo, para enfrentar la lógica de la urbanización neoliberal que se orienta a generar diferenciación social y fomentar la competitividad (Kipfer \& Keil, 2002; Smith, 2002). Asimismo, el alto perfil laboral de los habitantes favorece la autodefensa de sus intereses. Entre un grupo de 101 ciudades, Santa Mónica cuenta con mayor 
porcentaje de ocupación de hombres y mujeres que ejercen la abogacía; quinto lugar en arquitectos; sexto con mayor porcentaje de mujeres ocupadas en la industria de la computación, arte y entretenimiento; y séptimo en mujeres ocupadas en la industria de la publicidad, cine e industria de la grabación.

Los residentes de Santa Mónica practican una cultura asociativa. A pesar de poseer solo 21,7 kilómetros cuadrados de territorio y poco más de 90.000 habitantes, en 2010 existían al menos cuarenta organizaciones sin fines de lucro muy activas, pero hay cientos de ellas registradas en la ciudad (Great Nonprofits, 2013). Algunas impulsan la creación de una sociedad más sustentable, como Sustainable Works, una organización no gubernamental (ONG) de educación ambiental en escuelas y negocios que trabaja en coordinación con el Santa Monica College, el Center for a New American Dream y Green Seal. Actualmente existen nuevos movimientos civiles, vecinales y comunitarios para contrarrestar las políticas empresarialistas, como la Santa Monica Coalition for a Livable City (SMCLC) (alternativa a SMRR), cuya divisa es que "cualquier nuevo desarrollo en la ciudad no afecte la calidad de vida de sus habitantes, sino al contrario, que sirva a la comunidad" (sMcLC, 2013).

Las organizaciones vecinales son una base para la sustentabilidad urbana, y al gestionar las problemáticas de sus barrios contrapesan la política gubernamental; entre ellas se encuentran la Wilshire/Montana Coalition (Wilmont), Friends of Sunset Park, Ocean Park Association, Pico Neighborhood Association (PNA), North of Montana Association (nOMA), y la Mid-City Neighborhoods (MCN). Cada una tiene prioridades particulares a escala de la ciudad y del vecindario. Frente a esta propensión asociacionista, Soja (comunicación personal, 26 de mayo de 2010) destaca que esta ciudad fue capaz de sensibilizarse ante la problemática medioambiental con mayor rapidez que el resto de las localidades del área metropolitana de Los Ángeles. Sin embargo, advierte que el acento exagerado en la sustentabilidad que despliegan (que denomina también "eco-énfasis") tiende a alejar a las autoridades de Santa Mónica de las problemáticas sociales básicas, como la pobreza e indigencia.

En 1994, la coalición en el poder instituyó el plan de la ciudad sustentable -Santa Monica Sustainable City Plan-, que confiere a la sustentabilidad una naturaleza tridimensional, evaluando los logros a largo plazo a partir de tres formas de capital: i) capital natural (sus recursos naturales); ii) capital humano (perfil sociocultural de los residentes; capital social, vínculos entre las personas de la comunidad); iii) capital financiero y físico (infraestructura, bienes manufacturados, edificios, recursos de información, crédito y deuda). No obstante, al ceder a la lógica empresarialista y beneficiar al turismo y los bienes raíces, el gobierno privilegió lo financiero sobre lo social. Como respuesta han surgido nuevos movimientos de modestas dimensiones, espontáneos u organizados, que posicionan también nuevos temas en la agenda urbana para defender la sustentabilidad, creando una correlación de fuerzas sociales en disputa y negociación que influyen en la especificidad de la forma y el diseño urbano. La inconformidad con la política del gobierno local en el manejo de los asuntos sociales y los recursos está a flor de piel. En octubre de 2007 se formó la organización Treesavers (treesavers.blogspot.com) para luchar contra el reemplazo de más de cincuenta antiguos árboles ficus canopy de las calles segunda y cuarta, para colocar ginkgos (Treesavers, 2007). Rechazaron la versión oficial de 
que los ficus canopy eran insalvables y cuestionaron la medida como un intento por hacer más agradables esas dos calles para los turistas (Jacuot, 2009). Gracias a sus protestas evitaron que removieran del parque Douglas un árbol conocido como Australian bunya, con más de treinta años de antigüedad (Jarvey, 2009). Sin embargo, en 2008, veintitrés ficus no corrieron la misma suerte, al ser removidos como parte de un plan de mejoras al paisaje urbano. Con motivo del segundo aniversario de la "muerte de estos árboles", en 2010 los Treesavers salieron a las calles a protestar por tal agravio (Santa Monica Close-up, 2010). Estas réplicas ciudadanas a medidas oficiales alejadas de la sustentabilidad son frecuentes. Al igual que los Treesavers, los ciclistas, que sin constituir una asociación buscan mejoras para transportarse en un ambiente agradable y seguro, mantienen un contacto fluido con el gobierno. Aun cuando en 2009 Santa Mónica recibió el reconocimiento Bike Friendly Award (Premio a una ciudad amigable para el ciclista) por parte de la Liga de Ciclistas Americanos (League of American Bicyclists), los ciclistas locales no validan este galardón, pues exigen mejorar todavía más los espacios públicos (Cain, 2009), en especial en cuanto a la señalización y conectividad de las ciclovías, el establecimiento de bulevares para bicicletas y lugares seguros de estacionamiento (Kavanagh, 2010). Otro movimiento, el Ocean View Farms Community Garden (http://www. oceanviewfarms.net/), integrado con vecinos de Santa Mónica, Mar Vista y otras localidades del oeste de Los Ángeles, promueve jardines comunitarios y ofrece asesoría para el cultivo de plantas y el compostaje (Ocean View Farms, 2010).

Este activismo social no es aislado; se inscribe en el contexto de la posmetrópoli de Los Ángeles, que tiene una compleja división política territorial de 340 ciudades, 175 de ellas en el condado de Los Ángeles, colmada de contradicciones sociales, económicas y medioambientales que estimulan, pero también restringen, la unión formal e informal de sus residentes que buscan solucionar la problemática urbana que enfrentan.

La sustentabilidad en Santa Mónica incluye el despliegue de tecnologías sustentables, técnicas, estrategias y prácticas de planeación y diseño de la ciudad. Un compendio que epitomiza este dispositivo de instrumentos de planeación es el libro de John Lund Kriken (2010), City Building. Nine planning principles for the twenty first century.

El nuevo urbanismo, la certificación LEED y el crecimiento inteligente, sintetizan los fines impulsados por desarrolladores, ambientalistas, líderes políticos y grupos ciudadanos. Han logrado incorporarse, no sin controversias, en distintos planes, como el Land Use and Circulation Element (LuCE), el Santa Monica Sustainable City Plan, el Plan Específico para el Centro Cívico y el Plan Maestro para la Remodelación del Centro Comercial Santa Monica Place.

Bajo este urbanismo sustentable, el ayuntamiento instituyó la preservación de áreas naturales, la promoción del mantenimiento y construcción de parques, áreas verdes, espacios al aire libre de alta calidad para la salud, patrones de uso del suelo orientados a la heterogeneidad y diversidad de viviendas, regulaciones para la construcción y remodelación de edificios con materiales no contaminantes, medios alternativos de movilidad urbana a través de mejoras al transporte público y espacios amigables con ciclistas y peatones. Asimismo, propicia la acentuación de características distintivas de la ciudad con el fin de generar un "sentido de lugar" a través 
de un programa de preservación histórica, que alienta a los habitantes a asumir la conservación medioambiental como parte de la identidad local.

Los ciudadanos, a su vez, refuerzan su cultura de participación mediante la organización de talleres de discusión sobre políticas de planeación local, como ocurrió con los debates públicos realizados de 2004 a 2010 para la actualización del LuCE. Un tópico controversial fue la regulación de la altura de las construcciones, ya que la baja elevación de los edificios es parte distintiva del escenario urbano que brinda a los residentes un fuerte sentido de lugar. No obstante la oposición ciudadana, en junio de 2010 entró en vigor el LUCE autorizando, a propuesta de la Cámara de Comercio, un incremento de hasta 3,3 metros en la altura máxima de los edificios. Aunque el ayuntamiento argumentó fomentar "un diseño medioambientalmente más amigable", la medida fue cuestionada por grupos vecinales como estrategia empresarialista de uso del suelo que pone en riesgo el desarrollo a escala humana (Taborek, 2010).

Hay aspectos icónicos de la planeación sustentable que no son aplicables al territorio de Santa Mónica, como la densificación, por la falta de espacio para crecer y la oposición ciudadana a la construcción de edificios de gran altura. La dependencia del automóvil es otro problema, pues únicamente 16,7 por ciento de las personas que laboran en Santa Mónica reside ahí, mientras el resto se transporta a otras ciudades. Se requiere de grandes esfuerzos por parte de las autoridades de la megalópolis para tratar el problema, como la conclusión de una nueva línea del metro (Exposition Light Rail Transit Line), que se proyecta llegue a Santa Mónica en 2016.

Otro aspecto contradictorio de esta sustentabilidad es la injusticia social y espacial que conllevan sus proyectos de desarrollo urbano. Esto se patentiza en las afectaciones sufridas por el vecindario de Pico, donde se concentra la mayoría de la población latina. Pico posee 13.189 habitantes (16 por ciento del total de la ciudad) y alrededor de 5.600 viviendas. Comparado con el 13 por ciento de latinos en promedio que habitan en los otros barrios de la ciudad, este vecindario posee 38 por ciento de esa minoría. Tiene una base arrendataria más amplia (83 por ciento en Pico, 70,2 por ciento en el resto de la ciudad), un ingreso per cápita 5.000 dólares menor que el del resto de Santa Mónica y 22 por ciento de familias viviendo por debajo de la línea de pobreza, comparado con 5,4 por ciento del promedio local (City of Santa Monica, 2006). A pesar de estar relegado de los círculos de poder de la ciudad, Pico es uno de los barrios políticamente más activos. Forjó nuevos líderes, como Ana Jara y Óscar de la Torre, que se matricularon en la asociación Residentes de Santa Mónica en Defensa de los Derechos de Inquilinos (SMrR) para defender los derechos de las minorías locales.

$\mathrm{Al}$ encauzar esfuerzos en elevar la competitividad con el programa de la ciudad sustentable, el ayuntamiento ha descuidado asuntos prioritarios para la población de Pico, los cuales -según Ana Jara (comunicación personal, 27 de mayo de 2010) - son la creación de empleo, revitalización económica de la zona, educación de calidad, disminución de la violencia juvenil y protección de la salud pública. Frente a esto, el vecindario organiza, por su propia cuenta, acciones para asumir la problemática no atendida por el gobierno local, como lo hacen el Pico Youth and Family Center (Centro Juvenil y Familiar de Pico) y grupos religiosos para la 
prevención y disminución de la violencia juvenil. Otro ejemplo es la campaña a favor de la "justicia medioambiental", que se inició en junio de 2010 como respuesta a la desproporcionada distribución de los riesgos de la contaminación y los desechos tóxicos en la ciudad (Friedman, 2010).

Con una sustentabilidad acotada por objetivos económicos, la tradición de activismo ciudadano de Santa Mónica mantiene el empoderamiento de residentes movilizados por causas de justicia medioambiental, que asumen la contaminación como una forma de violencia que afecta la salud de los habitantes. Pero esta movilización no es compartida por todas las ciudades de la megalópolis de Los Ángeles, sino únicamente por aquellas zonas que aprovechan sus ventajas de aglomeración, como Santa Mónica, que se apoya en dichas ventajas para impulsar su crecimiento económico y atraer a grandes corporaciones. Como centro turístico de compras, desplegó con fuerza un marketing verde, que obstaculiza la aplicación de políticas efectivas de protección medioambiental y justicia social. El sector empresarial aprovecha la gestión medioambiental como estrategia de marketing urbano, con éxito. La remodelación del Centro Comercial Santa Monica Place es muestra de lo anterior; en su construcción se invirtieron 275 millones de dólares -sin subsidios de la ciudad-, se utilizaron materiales certificados como "verdes", generadores solares de electricidad, programas de eficiencia en el uso del agua e innovaciones en su diseño para el aprovechamiento de la luz y la ventilación natural. Este centro comercial ambientalmente amigable es promocionado como uno de los primeros equipamientos de este tipo de carácter sustentable en Estados Unidos y sus dueños esperan recuperar la inversión con el aumento de visitantes y consumidores.

En los últimos años, la noción de la sustentabilidad en Santa Mónica ha guiado la mejora física urbana para favorecer el turismo de compras. La elite que actúa como maquinaria de crecimiento local procura moldear el ambiente urbano para generar, con base en la idea de la sustentabilidad, un escenario cultural distintivo y paisajes simbólicos que promuevan una cultura de consumo conspicuo ético, creando asociaciones positivas con el acto de comprar, pues se hace en un "centro de consumo responsable" (Erickson \& Roberts, 1997; Goss, 1993).

\section{Conclusiones}

El desarrollo de Santa Mónica como ciudad sustentable es resultado de dos procesos contradictorios: la adopción de una política de planeación urbana con ideales progresistas, y el seguimiento de una estrategia de competitividad local dirigida por los empresarios y el gobierno local. Con el paso del tiempo, el Consejo de Gobierno, que provenía de movimientos ciudadanos de base, favoreció los intereses de la clase empresarial de la ciudad e instituyó un régimen desarrollista. La reorientación del discurso progresista, gracias al carácter y la tradición de activismo desde abajo, suscitó una serie de inconformidades ciudadanas que motivaron el surgimiento de movimientos de base -organizaciones vecinales, comunitarias y ONG- para hacer frente a las políticas que contravienen la visión cívica de la ciudad sustentable. Siguiendo la tradición pragmática estadounidense, el gobierno y los políticos locales han adecuado los principios de la sustentabilidad para acreditar sus decisiones y proyectos. 
Contrastando lo expuesto por Kriken (2010) y Farr (2008) sobre la sustentabilidad urbana, Santa Mónica muestra que una ciudad sustentable es algo más que un modelo de planeación, pues implica un cúmulo de ideales económicos, sociales y ambientales de desarrollo urbano que se articulan en un momento histórico determinado. Entendida de este modo, la sustentabilidad en la ciudad pasa de la ejecución de principios de planeación al concurso de complejos procesos de negociación en contextos espacio-temporales particulares, que permiten la aplicación de estrategias de desarrollo que -se espera- eleven el nivel de vida de la población y la calidad del medio natural y construido.

Santa Mónica muestra que el desarrollo sustentable es resultado de una permanente negociación entre los intereses y prioridades de los sectores que componen una comunidad urbana. Las presiones por mantener el dinamismo económico y la actividad turística, favoreciendo el mercado de bienes raíces, los precios de suelo y las aspiraciones de la clase empresarial, son fuerzas que empujan hacia una simulación de la sustentabilidad y al uso del discurso medioambiental con propósitos de lucro. La existencia de movimientos de base y la participación de ciudadanos seriamente comprometidos con los objetivos de cuidado medioambiental le han dado credibilidad al proyecto, generando ciertas formas reales de sustentabilidad urbana. No obstante los resultados obtenidos, persisten marcados vacíos en cuestiones de justicia social. OEURE

\section{Referencias bibliográficas}

Aguado, I.; Barrutia, J. \& Etxebarria, C. (2006). El desarrollo sostenible a lo largo del pensamiento económico. Memorias de la X Jornadas de Economia Crítica: ¿Alternativas al capitalismo? (1-32). Barcelona: Universidad Complutense.

Aguilar, L. (2006). Gobernanza y gestión pública. México, D.F.: Fondo de Cultura Económica.

Arendt, H. (1995). De la historia a la acción. Barcelona: Paidós Ibérica.

Barton, J. (2006). Sustentabilidad urbana como planificación estratégica. EURE, 32(96), $27-$ 45. doi: $10.4067 / S 0250-71612006000200003$

Beatley, T. (2001). Dutch green planning more reality than fiction. Journal of the American Planning Association, 67(1), 98-100. doi: 10.1080/01944360108976361

Bentivegna, V.; Curwell, S.; Deakin, M.; Lombardi, P.; Mitchell, G. \& Nijkamp P. (2002). A vision and methodology for integrated sustainable urban development. Building Research and Information, 30(2), 83-94. doi: 10.1080/096132102753436468

Benz, A. (2007). Governance in connected arenas - Political science analysis of coordination and control in complex rule systems. En D. Jansen (Ed.), New forms of governance in research organizations. Disciplinary approaches, interfaces and integration (pp. 1-22). Dordrecht: Springer.

Bithas, K. \& Christofakis, M. (2006). Environmentally sustainable cities: Critical review and operational conditions. Sustainable Development, 14(3), 177-189. doi: 10.1002/sd.262

Cain, C. (2009, julio 8). Cyclists circulating petition to strip city of bike friendly award. Santa Monica Daily Press. Recuperado de http://smdp.com/cyclists-circulating-petition-tostrip-city-of-bike-friendly-award/74658 
Castells, M. (2004). La cuestión urbana. México, D.F.: Siglo xxi Editores.

Community Service/Non profit Santa Monica. (23 de enero de 2010). About the Pico Neighborhood. Yelp: City of Santa Monica. http://www01.smgov.net/residents/sgv/PicoNeighborhood.htm

Deb, A. (1998). Sustainable cites in developing countries. Building Research and Information, 26(1), 17-28. doi: 10.1080/096132198370083

Ellis, C. (2002). The new urbanism: Critiques and rebuttals. Journal of Urban Design, 7(3), 261-291. doi: 10.1080/1357480022000039330

Erickson, B. \& Roberts, M. (1997). Marketing local identity. Journal of Urban Design, 2(1), 35-59. doi: 10.1080/13574809708724395

Farr, D. (2008). Sustainable urbanism: urban design with nature. Chicago, IL: Wiley.

Friedman, J. (2010, junio 6). Pico neighborhood activists call for Environmental Justice. The LookOut News. Recuperado de http://bit.ly/1bp33r8

Fulton, W. (1997). The reluctant metropolis: The politics of urban growth in Los Angeles. Los Angeles, CA: Solano Press Books.

Goss, J. (1993). The "magic of the mall": an analysis of form, function, and meaning in the contemporary retail built environment. Annals of the Association of American Geographers, 83(1), 18-47. doi: 10.1111/j.1467-8306.1993.tb01921.x

Great Nonprofits (2013). Santa Monica non profit and charities. Recuperado en julio de 2013, de http://greatnonprofits.org/city/Santa\%20Monica/CA

Harvey, D. (1989). The condition of posmodernity: an enquiry into the origins of cultural change. Nueva York, NY: Blackwell.

Harvey, D. (2007). Espacios del capital: Hacia una geografía crítica (C. Piña Aldao, Trad.). Madrid: Ediciones Akal.

Isin, E. (2002). Being political. Genealogies of citizenship. Minneapolis, MN: University of Minnesota Press.

Jacobs, J. (1971). La economía de las ciudades. Barcelona: Ediciones Península.

Jacquot, J. (2008). The battle of the Santa Monica ficus trees ends in defeat... for the trees. Treehugger. Recuperado en julio de 2013, en http://bit.ly/138RHoj

Jansen, D. (Ed.). (2007). Introduction. New forms of governance in research organizations. Disciplinary approaches, interfaces and integration (pp. xv-xix). Nueva York, NY: Springer.

Jarvey, N. (2009, agosto 11). After protests, city officials reconsider tree removal. Santa Monica Daily Press. Recuperado de http://bit.ly/1aqNmls

Kann, M. (1986). Middle-class radicalism in Santa Monica. Philadelphia: Temple University Press.

Kavanagh, G. (2010, 18 de marzo). Meeting with Santa Monica assistant city manager Jennifer Philips [Entrada blog]. Recuperado de http://bit.ly/a8h93V

Keil, R. (1998). Los Angeles: Globalization, urbanization and social struggle. Londres: Wiley.

Kipfer, S. \& Keil, R. (2002). Toronto Inc.? Planning the competitive city in New Toronto. Antipode, 34(2). doi: 227-264. 10.1111/1467-8330.00237

Kriken, J. (con Enquist, P.; Rapaport, R.). (2010). City building: Nine planning principles for the twenty-first century. Nueva York, NY: Princeton Architectural Press.

Larsen, K. (2005). New urbanism's role in inner-city neighborhood revitalization. Housing Studies, 20(5), 795-813. doi: 10.1080/02673030500214068

Lefebvre, H. (1972). La revolución urbana (M. Nolla, Trad.). Madrid: Alianza. 
Lieberherr-Gardiol, F. (2009). Urban sustainability and governance: issues for the twentyfirst century. International Social Science Journal, 59, 331-342. doi: 10.1111/j.14682451.2009.01670.x

Moffatt, I. (1999). Edinburgh: A sustainable city? International Journal of Sustainable Development \& World Ecology, 6(2), 135-148. doi: 10.1080/13504509909470002

Molotch, H. (1976). The city as a growth machine: toward a political economy of place. The American Journal of Sociology, 82(2) 309-332. URL estable: http://www.jstor.org/stable/2777096

Molotch, H.; Freudenburg, W. \& Paulsen, K. E. (1997). History repeats itself, but how? City character, urban traditional and the accomplishment of place. American Sociological Review, 65(6), 791-823. uRL estable: http://www.jstor.org/stable/2657514

Mumford, L. (2002). Historia natural de la urbanización (C. Jiménez, Trad.). Boletín $C+S$, 21. Recurso online. Disponible en http://habitat.aq.upm.es/boletin/n21/almum.html

Naess, P. (2001). Urban planning and sustainable development. European Planning Studies, 9(4), 503-524. doi: 10.1080/713666490

Nelson, L. (2001). Community sustainability and land use. Public Administration Review, 61(6), 741-746. doi: 10.1111/0033-3352.00144

O’ Connor, J. (2002). ¿Es posible el capitalismo sostenible?. En H. Alimonda (Ed.), Ecología, politica, naturaleza, sociedad y utopía (pp. 27-52). Buenos Aires: Consejo Latinoamericano de Ciencias Sociales (Clacso).

Ocean View Farma (enero de 2010). Ocean View: A non profit community garden in west Los Angeles. Recuperado en mayo de 2010, de http://www.oceanviewfarms.net/mission.html

Pal, L. (2006). Beyond policy analysis: public issue management in turbulent times. Toronto: Nelson Education.

Park, R. (1999). La ciudad y otros ensayos de ecología urbana. Barcelona: Ediciones del Serbal.

Pike, A.; Rodríguez Pose, A. \& Tomaney, J. (2011). Desarrollo local y regional. Valencia: Universidad de Valencia.

Pitt, L. (1997). Los Angeles A to Z: An encyclopaedia of the city and county. Los Angeles, CA: University of California Press.

Portney, K. E. (2003). Taking sustainable cities seriously: economic development, the environment, and the quality of life in American cities. Cambridge, MA: The мIT Press.

Riposa, G. (2004). Reinventing paradise: Santa Monica’s sustainable city program. PAQ, 28(2), 222-252. Disponible en http://bit.ly/1aqNZLN

Rykwert, J. (2002). The seduction of place. The history and future of the city. Nueva York, NY: Vintage Books.

Santa Monica (City). (2011). Quick facts from the US Census Bureau. Recuperado en mayo de 2013, de u.s. Census Bureau. http:/quickfacts.census.gov/qfd/states/06/0670000.html

Santa Monica Close-up (mayo 2010). In memoriam. Recuperado en julio de 2013, en http:// santamonicacloseup.com/photo-du-jour/2010/5/16/in-memoriam.html

Santa Monica Coalition for a Livable City (2013). Mission Statement. Recuperado en julio de 2013, en http://www.smclc.net/mission.html

Santa Monica Housing and Economic Development. (2008). Santa Monica Community Profile. Recuperado en julio de 2013, en http://www.smgov.net/Departments/HED/ eddContent.aspx?id=23577 
Santa Monica Rent Control Charter Amendment. (2012). Recuperado en julio de 2013, de http://bit.ly/11hHxR0

Santa Monica Sustainable City Plan. (2003). Recuperado en julio de 2013, de http://bit. ly/11hHnct

Schuppert, G. F. (2007). Governance reflected in political science and jurisprudence. En D. Jansen (Ed.), New forms of governance in research organizations. Disciplinary approaches, interfaces and integration (pp. 33-56). Dordrecht: Springer.

Scott, P. A. (2004). Santa Monica, a history on the edge. Mount Pleasant, sc: Arcadia Publishing.

Smith, S. (2002). New globalism, new urbanism: gentrification as global urban strategy. Antipode, 34(3), 427-450. doi: 10.1111/1467-8330.00249

Soja, E. (2000). Postmetrópolis: Critical studies of cities and regions. Oxford: Blackwell Publishers.

Souza, M. L. (2006). Social movements as 'critical urban planning' agents. City. 10(3), $327-$ 342. doi: 10.1080/13604810600982347

Swyngedouw, E. (1997). Neither Global Nor Local: 'Glocalization' and the Politics of Scale. En K. Cox (Ed.), Spaces of globalization: reasserting the power of the local (pp. 137-166). Nueva York/Londres: Guilford/Longman.

Taborek, N. (2010, mayo 20). Residents and chamber spar over LucE. Santa Monica Daily Press. Recuperado de http://smdp.com/residents-and-chamber-spar-over-luce/76526

Thornton, R. (marzo de 2011). sMRR's 1st Council Majority. Recuperado en julio de 2013, de http://www.smrr.org/news/NL221/FirstMajority.html

Treessavers. (2007). Save the trees in downtown Santa Monica! Recuperado en julio de 2013, de http://www.ipetitions.com/petition/saveourtrees/

un-Habitat - United Nations Human Settlements Programme. (2009). Planning sustainable cities: global report on human settlements 2009. Recuperado de http://bit.ly/IRnSu

Wheeler, S. (2003). The evolution of urban form in Portland and Toronto: implications for sustainability planning. Local Environment, 8(3), 317-336. doi: 10.1080/13549830306656

Whitehead, M. (2003). (Re)analyzing the sustainable city: nature, urbanization and the regulation of socio-environmental relations in the uk. Urban Studies, 40(7), 11831206. doi: 10.1080/0042098032000084550

\section{Documentos oficiales}

California Department of Finance. (2009, 1 de mayo). State Finance Department Releases New Population Data for 2008. Recuperado de http://bit.ly/15gitch

United States Census Bureau. (2011). Santa Monica (City) California (2011). Recuperado de http://1.usa.gov/vAtBhL 